\title{
Evaluation of Upper Limb Sense of Position in Healthy Individuals and Patients after Stroke
}

\author{
I. Cusmano, MEng ${ }^{1 *}$; I. Sterpi, PhD ${ }^{1}$; A. Mazzone, BIT ${ }^{2}$; S. Ramat, PhD ${ }^{3}$; \\ C. Delconte, $\mathrm{MS}^{4}$; F. Pisano, $\mathrm{MD}^{4}$ and R. Colombo, MEng ${ }^{1,2}$ \\ 1 "Salvatore Maugeri" Foundation, IRCCS, Service of Bioengineering, Pavia, Italy \\ 2 "Salvatore Maugeri" Foundation, IRCCS, Service of Bioengineering, \\ Veruno (NO), Italy \\ ${ }^{3}$ Department of Computer and Systems Science, University of Pavia, Pavia, Italy \\ 4 "Salvatore Maugeri" Foundation, IRCCS, Division of Neurology, Veruno (NO), Italy
}

Submitted July 2013. Accepted for publication December 2013.

\begin{abstract}
The aims of this study were to develop and evaluate reliability of a quantitative assessment tool for upper limb sense of position on the horizontal plane. We evaluated 15 healthy individuals (controls) and 9 stroke patients. A robotic device passively moved one arm of the blindfolded participant who had to actively move his/her opposite hand to the mirror location in the workspace. Upper-limb's position was evaluated by a digital camera. The position of the passive hand was compared with the active hand's 'mirror' position. Performance metrics were then computed to measure the mean absolute errors, error variability, spatial contraction/expansion, and systematic shifts. No significant differences were observed between dominant and nondominant active arms of controls. All performance parameters of the post-stroke group differed significantly from those of controls. This tool can provide a quantitative measure of upper limb sense of position, therefore allowing detection of changes due to rehabilitation.
\end{abstract}

Keywords: proprioception, sensory system, sense of position, neurorehabilitation, stroke

\section{INTRODUCTION}

Over the past few decades, studies on use-dependent plasticity have demonstrated that proprioceptive feedback plays a critical role in the reorganization process and subsequent recovery of the neuromotor system [1]. In the fields of physical therapy and rehabilitation, much attention is currently focused on sensory feedback acuity, in particular proprioceptive acuity, in both the clinical practice and laboratory. In fact, the sense of proprioception is considered a key source of feedback for promoting neural plasticity $[2,3]$. Proprioception can be defined as the ability of an individual to determine body segment position and movement in space [4-6]. It is based on sensory

*Corresponding author: Ivana Cusmano, "Salvatore Maugeri" Foundation, IRCCS, Service of Bioengineering, via Salvatore Maugeri 10, 27100 Pavia - Italy. Phone: (+39)0382592200. E-mail: ivana. cusmano@fsm.it. Other authors:irma.sterpi@fsm.it; alessandra.mazzone@fsm.it; stefano.ramat@unipv.it; carmen.delconte@fsm.it; fabrizio.pisano@fsm.it; roberto.colombo@fsm.it . 
signals that muscles, joints and skin receptors provide the central nervous system (CNS) consequent upon stretch and compression of body tissue. Because of the important role played by proprioception in maintaining posture and executing movement, patients who exhibit proprioceptive deficits cannot maintain their limbs in a steady posture or execute controlled movements in the absence of vision [7].

Proprioception on its own is difficult to measure; it is commonly evaluated by clinicians through tests which, however, have poor inter-rater reliability and sensitivity and give only a qualitative and subjective measure $[8,9]$. Different methods have been proposed for the quantitative evaluation of proprioceptive deficits $[1,7,10-20]$. Most of them rely on joint position-matching task procedures on a plane or, alternatively, the ipsilateral and contralateral matching of a joint angle [13]. They can be grouped into three broad categories: 1) those based on unimanual robotic devices [7, 11]; 2) those using bimanual robotic devices $[1,10,12,14,15]$; 3) those using motion analysis technologies [16-20]. The first type of method usually involves tasks of ipsilateral matching of a set of positions on a plane and requires participants to use memory to accurately match the target positions. The second type usually involves tasks in which the reference hand is positioned by one robot-arm and the subject is requested to actively move the other hand-arm (contralateral) in order to match the mirror position in space. This method eliminates the need for memory-based matching but requires expensive devices and involves greater inter-hemispheric communication compared with ipsilateral matching. The third category requires the use of simple or complex motion analysis systems and continuous assistance by a therapist in the case of contralateral matching tasks, and often the workspace tested is different from that involved in training.

In most of these tests, the patient is asked to re-create a reference position in the absence of vision. However, in some cases, proprioceptive acuity is assessed by judging the position of the hidden hand-arm under evaluation with respect to a visual reference target. In this case, one should be aware that the tested proprioception might be influenced by visual processes, in that a mis-localization of the visual reference position (due, for example, to a parallax error) could influence the matching performance. In all methods, accuracy of matching is subsequently measured by biomechanical equipment or through qualitative visual inspection by a trained operator. It is generally accepted that the magnitude of matching errors can constitute a useful estimate of proprioceptive acuity [1]. In other words, individuals who exhibit large position matching errors are considered to be proprioceptively impaired. Patients with large fiber neuropathy, for example, exhibit major deficits in motor control. These deficits include poor endpoint accuracy, reduced control of multisegmental dynamics, and inability to perform extended movement sequences. In addition, studies of human muscle spindle afferents have shown that attention can modify afferent signals. In particular, directing a subject's attention to the passive rotation occurring at a joint can cause changes in mean spike rate and functional dynamic range of the muscle spindle [21]. This suggests that proprioception may be modulated to provide a greater acuity for behaviourally significant limb movements. Such modulation may accompany motor learning, enhancing proprioception for newly learned motor tasks. In particular, recent findings 
have shown that proprioceptive acuity improves following motor learning but only in the region of the workspace explored during learning [22].

Modern neurorehabilitation is based on techniques of motor learning using advanced technologies such as robotics and virtual reality [23]. In general, during robot-aided rehabilitation, the device acts on the periphery, while learning takes place mostly in the high-level parts of the central nervous system. One third to one half of stroke patients (the population most commonly involved in studies using these rehabilitation technologies) exhibit proprioceptive deficits $[24,25]$. In addition, some reports indicate that intact position sense following stroke strongly correlates with motor recovery of the hemiplegic arm and predicts the extent of long-term motor recovery [26, 27].

In spite of its importance, the quantitative evaluation of position sense is not as widespread as expected. This is, on the one hand, because of the high complexity of systems using robot technologies that generally require bimanual devices and, on the other hand, because of the poor reliability of less complex systems. In addition, position sense impairment is a so-called 'body-related' disorder which, in general, is neglected in the clinical context because conventional rehabilitation programs usually focus on therapies targeted at recovery of the impaired limb. In most activities of daily living, the majority of object manipulations and interactions are performed at the centre of the workspace and involve bimanual tasks. Proprioception is crucial for coordinated movements; hence, the presence of proprioceptive deficits may have a great impact on activities of daily living [10, 28, 29]. For this reason, we hypothesized that using a bimanual protocol to assess proprioceptive performance could have some advantages over using a unimanual protocol. The number of devices available for robot training is increasing and their growing popularity is confirmed by the increased number of papers, studies and reports describing the application of robot technology to rehabilitation. Therefore, the development of a tool to evaluate proprioceptive deficits that uses unimanual devices already used for therapy in combination with a bimanual assessment protocol could be of clinical importance in that it would allow assessment of upper limb proprioception without the need for additional sophisticated technology.

The aims of this study were: a) to develop and validate a quantitative assessment tool for upper limb sense of position on the horizontal plane, by combining unimanual robot technology used in clinical practice with a low cost motion detection tool; b) to compare the difference in sense of position evaluated by this tool between healthy subjects and stroke patients.

\section{METHODS}

\subsection{Subjects}

The study was performed at the Salvatore Maugeri Foundation, IRCCS, Rehabilitation Institutes of Pavia and of Veruno (Italy). We evaluated 15 healthy subjects (control group) and 9 patients affected by sub-acute (less than 6 months from the acute event) and chronic stroke. The healthy subjects (female $=8$, male $=7$; mean age $32.7 \pm 11.5$ years) reported no history of visual, neurological or musculoskeletal disorders. Their handedness was evaluated through the Edinburgh inventory [30]. The 9 patients (female $=1$, male $=8$; mean age $61.8 \pm 1.3$ years) enrolled in this pilot study were those who, from 21 patients 
admitted with a diagnosis of stroke, were eligible and agreed to be enrolled. Inclusion criteria were the presence of a somatosensory deficit of the hemiparetic hand and the preservation of movement function in the contralateral limb. Exclusion criteria were the presence of elbow contractures, severe visual deficits, neglect syndrome, and limb apraxia. Patients were medically stable, had adequate comprehension of instructions and perceptual ability for testing. Table 1 reports clinical data of the patients enrolled.

Sensation was tested using parts of the Rivermead Assessment of Somatosensory Performance (RASP) [31,32]. Concerning proprioception, only the affected wrist and thumb were tested, through the full range of motion. Patients, with eyes closed, underwent 6 trials of passive flexion and extension of each joint, and were asked to indicate when they felt the joint moving and in what direction.

In addition, touch of the palm and thumb of the affected hand were tested (with the patients' eyes closed). Subjects were asked to indicate when they felt their hand (lightly) touched and where. Based on the number of correct responses, the sensation for each modality at each anatomical site was classified as follows: score $0-1=$ absent, 2-4 = impaired, 5-6 = normal.

The study was carried out in conformity with the Declaration of Helsinki of the World Medical Association; all participants gave their informed consent to participate in the study, which had been approved by the local scientific and ethics committees.

\subsection{Evaluation Apparatus}

The apparatus for quantitative evaluation of upper limb sense of position on the horizontal plane consisted in a 2-degree of freedom robotic device, "Braccio di Ferro", combined with a low cost motion analysis system composed of a standard digital camera (Sony ${ }^{\circledR}$, DCR-PC1000E) [33] (Figure 1a). In order to enable the position of both arms to be evaluated, the camera was mounted on an adjustable support overhanging the robot workspace at a distance of about $1.2 \mathrm{~m}$ from the horizontal plane at which motor tasks were executed. This way, the camera could

Table 1. Clinical characteristics of the post-stroke patients enrolled in the study.

\begin{tabular}{|c|c|c|c|c|c|c|c|}
\hline ID & ender & $\begin{array}{c}\text { Time since } \\
\text { acute event } \\
\text { (months) }\end{array}$ & $\begin{array}{l}\text { Stroke } \\
\text { Type }\end{array}$ & $\begin{array}{l}\text { Hemisphere } \\
\text { Lesion }\end{array}$ & FIM & $\begin{array}{r}\text { Touch } \\
\text { Sensation }\end{array}$ & Proprioception \\
\hline 1 & $\mathrm{~F}$ & 36 & hemorrhagic & right & 74 & absent & impaired \\
\hline 2 & M & 6 & ischemic & right & 32 & absent & absent \\
\hline 3 & M & 24 & ischemic & left & 119 & normal & impaired \\
\hline 4 & M & 120 & hemorrhagic & left & 108 & absent & impaired \\
\hline 5 & M & 4 & hemorrhagic & right & 105 & impaired & normal \\
\hline 6 & M & 3 & ischemic & left & 61 & normal & impaired \\
\hline 7 & M & 60 & hemorrhagic & left & 113 & absent & impaired \\
\hline 8 & M & 3 & ischemic & left & 86 & impaired & normal \\
\hline 9 & M & 12 & ischemic & left & 111 & absent & impaired \\
\hline
\end{tabular}



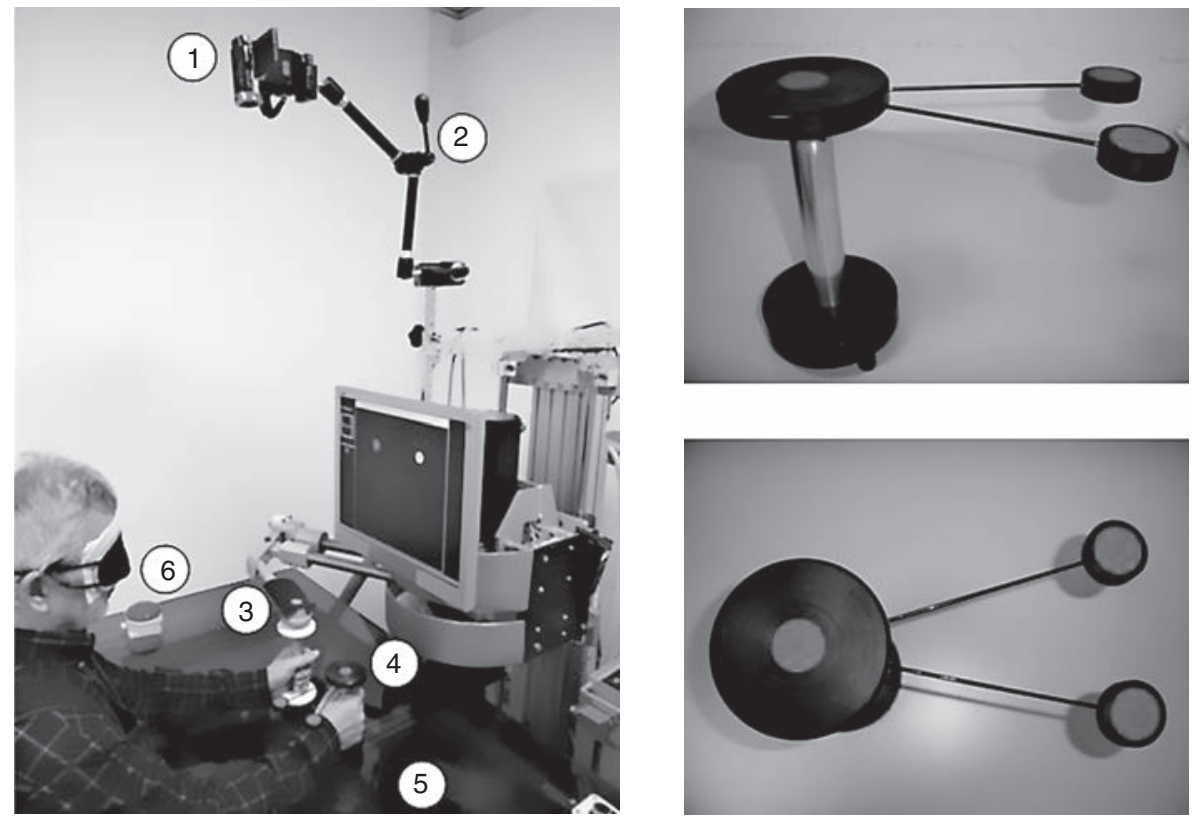

Figure 1. Experimental setup. Panel a) shows the robotic device "Braccio di Ferro" including the digital camera (1), with its articulated adjustable support (2), the robot arm and handle (3) used to move the passive arm, the active handle (4) whose particular configuration is highlighted in the two pictures of panel b), the robot desk (5) and a safety switch (6) to stop robot movements in case of problems.

frame a workspace of $80 \mathrm{~cm} \times 40 \mathrm{~cm}$. The end-effector of the robot apparatus consisted of a sensorized handle which was grasped by the patient and moved through the workspace of the device (i.e., on the horizontal plane). Subjects were comfortably seated at the robot desk in such a way that their mid-sagittal axis was approximately aligned with the centre of the workspace, with their trunk fastened to the seat back by a special jacket to avoid trunk movements.

During the test, both limbs of the subject were supported by the robot desk. Upper limb positions were measured by means of two handles grasped by subjects. The first one, called the "passive handle", was the robot handle that passively moved one of the subject's arms and hand (in healthy subjects, the left or right limb; in stroke patients, the impaired limb) in a predefined set of workspace positions. The handle had a coloured marker in the centre and was moved by the robot at low speed $(<30 \mathrm{deg} / \mathrm{s})$ so as not to induce reactions in the presence of spasticity of the impaired limb. If the patient was unable to grasp the passive handle, the impaired hand was fastened to the robot-handle by a special strap. The second one, called "active 
handle" (Figure 1b), was similar in size and weight to that of the robot device and free to be moved in the workspace (not connected to the robot). In order to provide similar haptic sensation during movement, the handle was supported by three lowfriction pads. It was actively moved by the subject using the contralateral arm (with respect to the robot-connected arm). The active handle had three coloured markers: one placed on the handle centre, the other two placed at $10 \mathrm{~cm}$ from the centre forming a fixed triangular geometry. This particular configuration was chosen to allow detection of the position of the active handle also in situations where the robot arm, because of its mechanical configuration, was covering the central marker. This way, the active handle could be freely moved below the robot arm. As a consequence, the marker placed on the passive handle was at a higher level than that of the active handle thus introducing a parallax problem in the position detection system. The system provided conversion from pixels (of the camera image) to millimetres, through a specific algorithm compensating for parallax errors, thanks to a calibration procedure using a 41-marker calibrated grid. During calibration, the markers of the active and passive handles were subsequently aligned with each point of the reference grid using a laser beam, thus allowing the mapping of pixels to millimetres in known positions of the workspace. The images captured by the camera were processed so as to obtain identification and classification of the markers. Then, the Cartesian coordinates in $\mathrm{mm}$ of the centre of a marker placed at a generic point on the horizontal plane were calculated from the pixel coordinates through a processing algorithm based on a neural network conversion procedure. Due to the limited number of known data which composed the training set (the calibrated grid), an exact Radial Basis Function Neural Network was chosen [34]. Both the image processing and neural network conversion procedure were implemented using Matlab $^{\circledR}$ custom software (The Math Works, Natick, Massachusetts, USA). The calibration procedure provided a maximum error between actual and estimated positions of $1.2 \mathrm{~mm}$ (spatial resolution).

\subsection{Experimental Protocol}

\subsubsection{Mid-Sagittal Axis Identification}

All subjects were given detailed instructions about how the evaluation would be carried out. In order to allow easy identification of the workspace symmetry axis independent of subjects' position at the robot desk, they were requested to perform a short exercise during which the robotic device passively moved the attached upper limb to one of three spatial locations (targets) on a vertical axis spaced $10 \mathrm{~cm}$ apart. With the assistance of visual feedback (eyes open), subjects then actively moved the other hand to the mirror location in space (Figure 2a). After movement completion, the examiner acquired a picture of the two handles.

This task was repeated three times for each of the three targets, for a total of 9 movements. The mid-point of the segment connecting each point reached by the passive $\operatorname{arm}\left(\mathrm{P}_{\mathrm{i}}\right)$ to the respective point reached by the active $\operatorname{arm}\left(\mathrm{A}_{\mathrm{i}}\right)$ was considered as a point lying on the mid-sagittal axis $\left(\mathrm{MSA}_{\mathrm{i}}\right)$. The mean value of $\mathrm{x}$-coordinates (lateral direction) 
(a)

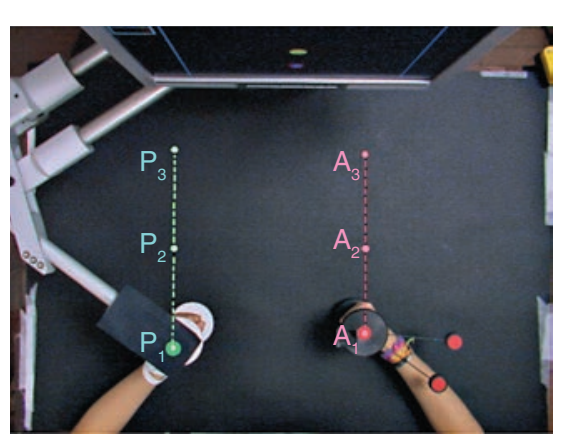

(b)

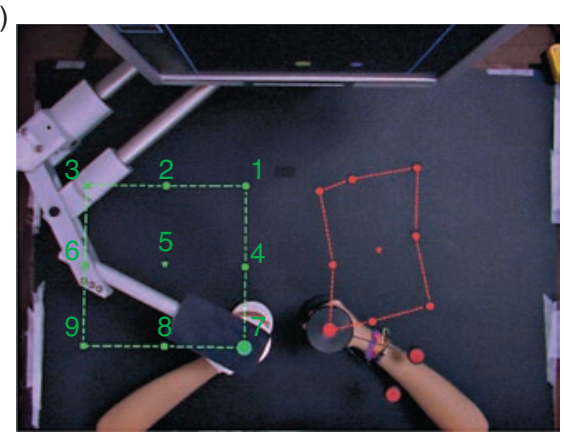

Figure 2. Active and passive hands positioned in the workspace, framed by the camera. (a) Exercise to search mid-sagittal axis with, in evidence, the three points where the passive hand moves $\left(\mathrm{P}_{\mathrm{i}}\right)$ and the three points where the active hand moves $\left(\mathrm{A}_{\mathrm{i}}\right)$. The red circles show the triangular geometry formed by the active handle's markers. (b) Exercise for sense of position evaluation; on the left are the target positions reached by the robotic device (numbered from 1 to 9) and on the right the mirror positions reached actively by a typical patient.

of the $\mathrm{MSA}_{\mathrm{i}}$ points allowed the identification of the mid-sagittal axis, that served as symmetry axis for the following task. It was different for each subject, depending on the subject's arm length and body position.

\subsubsection{Evaluation Task}

After this task, subjects were blindfolded with a black tissue mask in order to impede use of visual feedback, and underwent the evaluation task. The robotic device performed a pre-defined reaching sequence, moving the end-effector in a $20 \mathrm{~cm} \times 20 \mathrm{~cm}$ area in the half-workspace of the passive arm. In particular, subjects were requested to grasp the robotic handle with the passive hand, to relax the limb and let the robot move it to one of 9 different spatial locations (Figure 2b). Once the desired position was reached, the robot stopped moving for about 8 seconds and subjects were asked to move their opposite (active) hand and handle to the mirror location in space. If subjects required more time to reposition their arm, the 8-sec period between two tasks was increased. After stable positioning, visually assessed by observation of the active handle targets, the examiner took a picture of the workspace with the digital camera. The robot then resumed action, moving the passive arm to the next target starting from the last reached position. The sequence of reaching positions was randomized so as to obtain 6 positionings for each target for a total of $54(6 \times 9)$ reaching tasks. In healthy controls, the protocol was executed both with the dominant and non-dominant active arm in a pseudo-randomized order across 
subjects. Evaluations of the first and second limb were separated by a 10 -min resting period. The complete test for each limb lasted about 20 minutes.

\subsection{Evaluation Parameters}

The performance on matching was quantified by measuring the Cartesian position of the centre of the handles grasped by subjects and detected in each captured image. In order to compare actual (active) and desired (passive) hand positions, the raw positions of the passive hand were artificially mirrored across the $\mathrm{x}$-coordinate in correspondence to the pre-determined mid-sagittal axis. This way, the actual and desired positions would overlap in the case of perfect matching. In accordance with Dukelow et al. [10], for each subject, raw data were processed in order to compute the following quantitative parameters:

- Mean Error: the mean value of the 54 error values, i.e., the mean value of the absolute differences between the actual and the desired positions, respectively. This was a global parameter indicating simply the presence of proprioceptive deficits.

- Variability: to describe reaching-to-reaching consistency of the active hand positioning. Variability was obtained by computing the standard deviation of the active-hand positions for each spatial location. Then we computed the mean value of the standard deviations for the 9 target locations in the $\mathrm{x}$ coordinate $\left(\operatorname{var}_{\mathrm{x}}\right)$; note that var stands for variability and not for variance), ycoordinate $\left(\operatorname{var}_{\mathrm{y}}\right)$, and the linear variability for both coordinates combined $\left(\operatorname{var}_{\mathrm{xy}}\right)$ as the following:

$$
\operatorname{var}_{x y}=\sqrt{\operatorname{var}_{x}^{2}+\operatorname{var}_{y}^{2}}
$$

- Spatial contraction/expansion: designed to describe the range/area of the workspace matched by the active hand relative to that of the passive hand. Spatial contraction/expansion along the $\mathrm{x}$-axis $($ cont/exp $\mathrm{x}$ ) was obtained by computing the difference $\left(\right.$ range $_{\mathrm{x}}$ ) between the mean $\mathrm{x}$ positions for the 3 rightmost and the 3 leftmost targets reached by the active hand as compared with the passive one using the following equation:

$$
\text { cont } / \exp _{x}=\frac{\text { range }_{x_{\_} \text {active }}}{\text { range }_{x_{-} \text {passive }}}
$$

Values below 1 were obtained when the range of space explored by the active hand was smaller than that obtained by the passive hand, thus indicating a spatial contraction. Conversely, spatial expansion was indicated by values above 1 . A similar procedure was performed to compute contraction/expansion along the yaxis $\left(\right.$ cont/exp $\mathrm{y}_{\mathrm{y}}$. Spatial contraction/expansion along both coordinates (cont/exp $\mathrm{xy}_{\mathrm{xy}}$ ) was obtained by computing the area spanned by the active hand for the 8 peripheral targets. This value was then normalized to the total spatial area spanned by the passive hand. The spatial contraction/expansion parameters are expressed in normalized arbitrary units. 
- Systematic shifts: the constant errors between the active and passive hands. They were obtained by computing the mean error between the active and passive hand for each spatial location, and calculating the mean value for all target locations. Systematic shifts were obtained for the $\mathrm{x}$-coordinate $\left(\mathrm{shift}_{\mathrm{x}}\right)$, y-coordinate $\left(\right.$ shift $\left._{\mathrm{y}}\right)$ and both coordinates $\left(\right.$ shift $\left._{\mathrm{xy}}\right)$ by the following equation:

$$
\operatorname{shift}_{x y}=\sqrt{\operatorname{shift}_{x}^{2}+\operatorname{shift}_{y}^{2}}
$$

\subsection{Statistical Analysis}

Test-retest reliability analysis was conducted on a subset of 14 subjects ( 8 normal subjects and 6 pathological subjects) who were evaluated on two consecutive days. We first conducted a repeated measures ANOVA to verify that there were no systematic errors between the two sets of data. Then the Intraclass Correlation Coefficient (ICC) and the Standard Error of Measurement (SEM) were computed. In particular, ICC was computed according to the McGraw and Wong two ways fixed model (C,1 equation) [35]. It was defined as the proportion of variance of interest (variance among subjects) related to the total variance [36]. It ranges from 0 (poor reliability) to 1 (excellent reliability). SEM is expressed in the metric units of the measured parameters and includes both random and systematic components of measurement error [36].

In order to assess dependency of sense of position on handedness, control subjects executed the test twice: once using the dominant arm as the active arm and the other time using actively the non-dominant arm. To this end, the distribution of variability for the three main parameters $\left(\mathrm{var}_{\mathrm{xy}}, \operatorname{shift}_{\mathrm{xy}}\right.$, cont/exp $\mathrm{xy}$ ) was analysed by means of the Kolmogorov-Smirnov test. To evaluate if target positions could influence the parameters with respect to their distance from the subject's body and mid-sagittal axis, the ANOVA test (unpaired data) was carried out for the mean error, shift and variability parameters. In particular, we analysed the comparisons NEAR (targets 7,9) vs. FAR (targets 1,3) from body and NEAR (targets 1, 7) vs. FAR (targets 3, 9) from mid-sagittal axis. In practice, for this evaluation, only the positions of the vertex of the square (Figure $2 b$ ) were considered in the analysis.

Student's unpaired t-test was conducted to assess the ability of the parameters to distinguish between normal and pathological subjects.

A significance level of 0.05 was adopted for all statistical tests. The Bonferroni correction was applied to adjust the significance level for multiple comparisons. Statistical analysis was performed using the StatView statistical package (SAS Inst., NC-USA). Calculations of ICC and SEM were implemented using the Matlab development environment (The Math Works, Natick, Massachusetts, USA).

\section{RESULTS}

\subsection{Test-Retest Reliability}

Thirteen out of the 14 subjects ( 8 controls and 5 patients) who were part of the testretest reliability analysis subgroup completed the protocol. The repeated measures analysis of variance (ANOVA), performed for each variable to rule out a possible 
systematic error between the two consecutive days of trial, revealed no significant systematic error for any parameter. Table 2 presents the ICC and SEM values for all parameters. Specifically, ICC calculated for Contr/Exp $\mathrm{xy}_{\mathrm{y}}$ was $0.84(\mathrm{p}<0.001)$, indicating excellent test-retest reliability. Good reliability was also observed for the other parameters, with the ICC being equal to $0.78(\mathrm{p}<0.001)$ for Error $_{\mathrm{xy}}, 0.81$ ( $\mathrm{p}<$ $0.001)$ for $\operatorname{Var}_{x y}$ and $0.72(p<0.01)$ for Shift $x y$. The SEM for Error ${ }_{x y}, \operatorname{Var}_{x y}$, Shift Shy and Contr/Exp xy $_{\text {wa }} 1.6,0.6,1.9 \mathrm{~cm}$, and 0.16 (normalized units), respectively.

\subsection{Individual Subject Examples}

Each subject's performance was graphically summarized by reporting, for each target location, the passive hand's mean position mirrored across the x-coordinate with respect to the mid-sagittal axis and the mean position assumed by the active hand.

In addition, the confidence ellipse representing one standard deviation was overlapped to indicate within-subject variability. These plots provided a simple, clear representation of the subject's performance. Figure 3a reports the typical behaviour of a normal subject. No significant shift, contraction or expansion is evident in the plot. Further, a small variability can be observed. The remaining plots represent three different variability patterns obtained in three patients. In particular, they illustrate marked variability (Figure 3b), shift (Figure 3c) and contraction (Figure 3d) patterns.

\subsection{Control Group Analysis}

Only one out of 15 healthy controls was left-dominant. No difference was observed in controls between their dominant and non-dominant active arms. In particular no significant difference was found either for variability ( $\operatorname{var}_{\mathrm{xy}} ; \mathrm{P}=0.89$ ), shift ( shift $_{\mathrm{xy}} ; \mathrm{P}$ $=0.31)$ or contraction/expansion $\left(\operatorname{cont} / \exp _{\mathrm{xy}} ; \mathrm{P}=0.14\right)$. Therefore, dominant and

Table 2. ICC and SEM values evaluating the test-retest reliability of the parameters assessing sense of position

\begin{tabular}{llc}
\hline Parameter & ICC & SEM \\
\hline $\operatorname{Var}_{\mathbf{x}}(\mathbf{c m})$ & 0.82 & 0.58 \\
$\operatorname{Var}_{\mathbf{y}}(\mathbf{c m})$ & 0.69 & 0.40 \\
$\operatorname{Var}_{\mathbf{x y}}(\mathbf{c m})$ & 0.81 & 0.61 \\
Shift $_{\mathbf{x}}(\mathrm{cm})$ & 0.77 & 2.36 \\
Shift $_{\mathbf{y}}(\mathbf{c m})$ & 0.95 & 0.71 \\
Shift $_{\mathbf{x y}}(\mathbf{c m})$ & 0.72 & 1.91 \\
Cont$_{\mathbf{E x p}}$ & 0.85 & 0.12 \\
$\operatorname{Cont}_{\mathbf{E x p}}$ & 0.95 & 0.07 \\
$\operatorname{Cont}_{\mathbf{E x p}}$ & 0.84 & 0.16 \\
Error $_{\mathbf{x}}(\mathbf{c m})$ & 0.70 & 1.53 \\
Error $_{\mathbf{y}}(\mathbf{c m})$ & 0.93 & 0.61 \\
Error $_{\mathbf{x y}}(\mathbf{c m})$ & 0.78 & 1.61
\end{tabular}


(a)

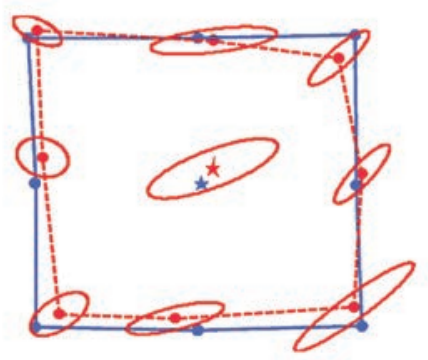

(c) $\quad \mathrm{ID}=6$

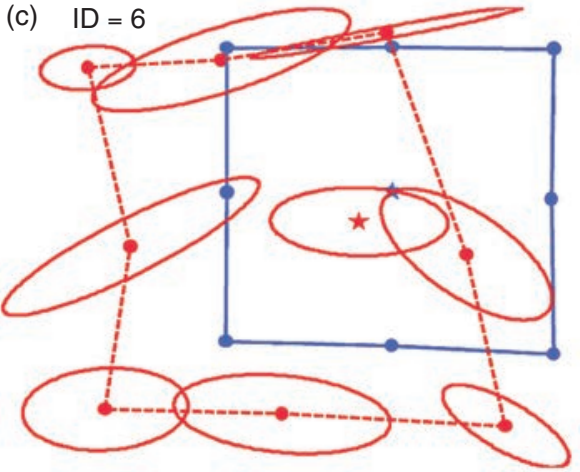

(b)

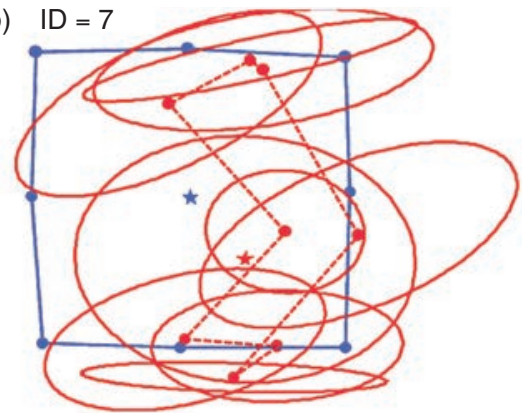

(d)

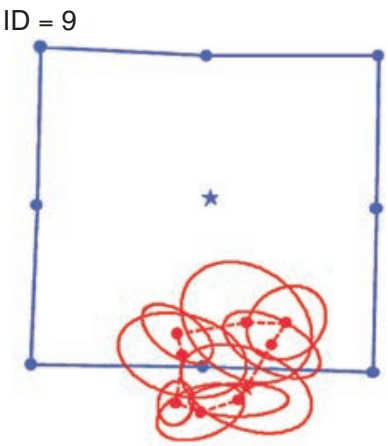

Figure 3. Data representing 4 different typical behaviours: (a) normal subject, (b) patient showing high variability, (c) patient showing shift of workspace, (d) patient showing a marked workspace contraction. Blue target and lines represent data from the passive hand mirrored across the $\mathrm{x}$-coordinate. Red target and lines represent correspondent mean positions reached by the subject's active arm. Ellipses around the targets represent one standard deviation within-subject variability.

non-dominant arm data were grouped together so as to constitute the normative data to be used for comparison with patients.

Figure 4 presents the mean values and standard deviations of the evaluation parameters measured in the control group. One can note that the spatial contraction/expansion parameter along the $\mathrm{x}$-axis $\left(\operatorname{cont} / \mathrm{exp}_{\mathrm{x}}\right)$ and consequently the cont/exp $p_{x y}$ were smaller than 1 , indicating a slight perceived spatial contraction. In addition, they showed a higher standard deviation than that obtained for the same parameter along the $\mathrm{y}$-axis $\left(\operatorname{cont} / \exp _{\mathrm{y}}\right)$.

Table 3 exhibits the results of the ANOVA test evaluating the influence of target position on the parameters with respect to their distance from the subject's body and mid-sagittal axis. In particular, the $\operatorname{var}_{\mathrm{x}}, \operatorname{var}_{\mathrm{y}}, \operatorname{var}_{\mathrm{xy}}$, $\operatorname{shift}_{\mathrm{y}}$ and error $_{\mathrm{y}}$ values were 

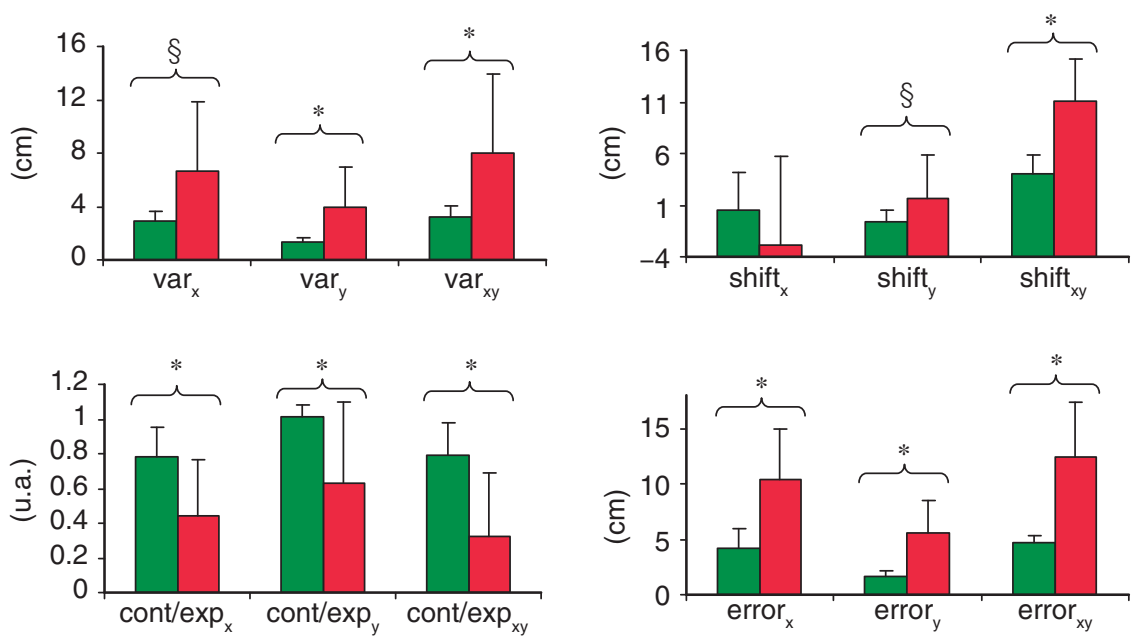

Control subjects $(n=15) \square$ Post-stroke patients $(n=9)$

Figure 4. Mean values (green and red bars) and standard deviations (lines on the bars) of the parameters evaluating sense of position in control subjects and post-stroke patients, and results of statistical analysis of their comparison. $(\S \mathrm{p}<0.01, * \mathrm{p}<0.001)$.

Table 3. Statistical analysis regarding the influence of mid-sagittal axis and body distance from targets on the parameters evaluating sense of position

(ANOVA p-value)

\begin{tabular}{lccc}
\hline Parameter & $\begin{array}{c}\text { Near vs. Far from } \\
\text { Mid-Sagittal Axis }\end{array}$ & $\begin{array}{c}\text { Near vs. Far from } \\
\text { Body }\end{array}$ & $\begin{array}{c}\text { Interaction } \\
\text { Mid-Sagittal Axis } \\
\text { \& Body }\end{array}$ \\
\hline Var $_{\mathrm{x}}$ & $<0.001$ & 0.020 & 0.170 \\
Var $_{\mathrm{y}}$ & $<0.001$ & 0.090 & 0.440 \\
Vary $_{\mathrm{xy}}$ & $<0.001$ & 0.020 & 0.340 \\
Shift $_{\mathrm{x}}$ & 0.680 & 0.280 & 0.690 \\
Shift $_{\mathrm{y}}$ & 0.002 & 0.320 & 0.890 \\
Shift $_{\mathrm{xy}}$ & 0.250 & 0.850 & 0.610 \\
Error $_{\mathrm{x}}$ & 0.320 & 0.580 & 0.980 \\
Error $_{\mathrm{y}}$ & $<0.001$ & 0.710 & 0.300 \\
Error $_{\mathrm{xy}}$ & 0.066 & 0.610 & 0.710
\end{tabular}


significantly higher for targets far from the mid-sagittal axis. Only $\operatorname{var}_{\mathrm{x}}$ and $\operatorname{var}_{\mathrm{xy}}$ were significantly higher for targets far from the body. No significant interaction was observed between the distance from mid-sagittal axis and that from body for any parameter. In other words, the parameters evaluating sense of position were influenced by the target distance from the mid-sagittal axis but not by that from the subject's body.

\subsection{Patient Group Analysis}

Figure 4 presents the mean values and standard deviations for the parameters obtained in the post-stroke patients and the $\mathrm{p}$ value for their comparison with the controls. All parameters, excepting shift $x$ values, were significantly higher in patients than controls. Most patients demonstrated the phenomenon of spatial contraction $\left(\right.$ cont/ $\left.\exp _{\mathrm{xy}}<1\right)$, indicating that their active arm moved into a smaller area of the workspace than the passive arm. Only one patient showed spatial expansion along both axes (cont/exp $\mathrm{x}_{\mathrm{xy}}>1$ ); none presented spatial expansion along only the $\mathrm{x}$-axis $\left(\operatorname{cont} / \exp _{\mathrm{x}}>1\right)$ and only 3 patients exhibited spatial expansion along the $y$-axis $\left(\right.$ cont $\left./ \exp _{\mathrm{y}}>1\right)$.

\section{DISCUSSION}

The main purpose of this study was to develop and validate a quantitative assessment tool for upper limb sense of position on the horizontal plane. Our results for control and pathological subjects are generally in line with those obtained by Dukelow et al. [10] using a bimanual device. However, likely because our control group had younger subjects than those enrolled in the Dukelow et al. study, the parameters' ranges of variation were smaller than those in their study. On the other hand, the parameters' ranges of variation for stroke patients were quite similar between the two studies. We showed that the sense of position in normal subjects is independent of their handedness. The left-arm advantage for position matching is well known, and is likely due to an enhanced proprioceptive feedback processing of the right-hemisphere. Therefore, individuals with right- versus left-hemisphere injury may be expected to exhibit greater proprioceptive deficits. In our study, hand dominance was considered only in healthy controls in order to verify if we could merge together data of their dominant and non-dominant arm. Obviously, stroke patients' sense of position can be evaluated only in the unimpaired arm, dominant or non-dominant, depending on the site of the brain lesion and dominant hemisphere, and clinicians are generally interested in evaluating changes of this measure due to rehabilitation rather than the absolute error values.

The present tool was able to discriminate between healthy and pathological conditions and it allowed to detect and quantify the proprioceptive deficits. It is worth noting that our system, in contrast to others, is based on the same type of device (unimanual) used for robot-assisted therapy, thus enabling a more widespread use of this tool for the quantitative evaluation of sense of position. 
Although joint movements and muscle actions are closely related to external stimuli from the environment, such as gravity and external forces, the tool we developed involved movements on the horizontal plane with arm support, and hence without the influence of gravity. In addition, proprioception is the awareness not only of the position but also of movement kinematics and kinetics. Thus, even if our tool can provide only an incomplete evaluation of proprioception, from a clinical perspective, it should be considered as a practical compromise between an easy-to-perform evaluation and a more detailed, time-consuming evaluation. Actually one might suppose that the placement of portable electrogoniometers on both upper extremities also would allow estimations of the impairment of the sense of position. This in general would be practical mainly for elbow joints using simultaneous multi-planar motions of both upper limbs. Conversely, the use of other motion analysis techniques such as magnetometers and inertial sensors could allow more sophisticated assessment protocols to be implemented. The system we developed allows an easy evaluation in different positions of the robot workspace, involving the displacement of multiple joints (i.e., wrist, elbow and shoulder) at the same time. Of course, the position of each joint can influence the measurement of the sense of position. However, although the parameters we computed should be considered as global parameters, providing measurement of the whole limb performance, their values in specific directions $(\mathrm{x}, \mathrm{y})$ may help in relating performance to specific postures. In addition, evaluation is carried out in the same workspace in which patients usually practice sensorimotor training, and this should allow the assessment of sensorimotor training effects on the reduction of proprioceptive deficits and, possibly, the development of specific rehabilitation protocols devoted for this purpose.

Recent literature has demonstrated that proprioceptive acuity is not uniform across the explored workspace [7]. Specifically, the estimation of arm position in different regions of the workspace is related to limb geometry [16,37-39] and to the integration of different information from different tasks by the CNS [15]. It is known that the reliability of information about perceived limb position may vary with the magnitude of upper limb joint angles, and with a more reliable perception pertaining to positions proximal to the human body $[40,41]$. In practice, more extreme joint positions are usually overestimated; this may be due to peripheral sensory signals biasing estimates as a safety mechanism to prevent injury [15]. Particularly, shoulder and elbow angles are closely interconnected, and it has been demonstrated that humans over-estimate their elbow extension with shoulder abduction but not during adduction. In other words, elbow angle is misperceived when the shoulder is abducted [15]. This fact should justify the contr/exp parameter $<1$ observed in our group of normal subjects. Moreover, the dependence of the variability parameter on distance from the mid-sagittal axis observed in our study could be therefore related to the increased shoulder abduction characterizing the more distal positions. Conversely, we did not observe any variability increment in radial direction with respect to the body front. This is consistent with van Beers [16], who showed that, during a localization task on the horizontal plane, proprioception in the radial 
direction with respect to the shoulder is more precise than localization in the lateral direction. In particular, if the distance from the shoulder decreased (i.e., smaller variance), localization of hand positions was more precise.

Two patients did not exhibit proprioceptive deficits in the clinical assessment of proprioception (ID $=5$, ID $=8$ ) and they had variability values slightly outside the normality ranges and a markedly contracted workspace. Two other $(I D=3, I D=10)$ patients were classified by the clinician as having an evident sense of position deficit but their variability was not significantly different from that of healthy subjects. First of all, a clinical evaluation including also testing of elbow and shoulder joints would have been beneficial; in addition, this could be ascribed to the ambiguous nature of the variability parameter, that on its own is not able to clearly indicate deficits. In fact, we observed that patients with important proprioceptive deficits tended to move their active arm in a reduced portion of space; consequently they had a reduced variability. We can hypothesize that a failure of the compromised arm to perceive the real amplitude of passive movements would be reflected in an underestimation of the workspace to be explored with the active arm. In this light, the same hypothesis could explain why the index of contraction/expansion resulted $<1$ in most of our patients, thereby indicating a contraction that was once again due to a reduced perception of the workspace to be explored. In other words, subjects who cannot perceive the passive movement of the compromised arm move their active (not compromised) arm in a limited region of the workspace. As a result, their variability is reduced and the active area is contracted and shifted. In addition, the standard deviation of several parameters, especially in stroke patients, was large. This can be explained by the fact that different patients exhibit different patterns of performance; for example, as shown in figure 3, that some patients exhibited workspace contraction, others a marked shift, etc. Therefore, with the prospect of using this evaluation method as a diagnostic tool, it is desirable that future studies address the computation of the minimal clinically important difference (MCID), i.e., the value indicating a real and clinically significant change of the parameters. Our results indicate that this should be done by computing different values for the diverse patterns of performance (e.g., marked shift, contraction/expansion, etc.)

Limitations of the present study include the small sample size as regards both healthy controls and patients. For this reason, the study should be considered as preliminary. In order to fully address the reliability of this tool, a future study with a larger sample of subjects divided into different classes of age and gender needs to be conducted. Further, it has been shown that proprioceptive performance differences in position and motion sense clearly exist between the young and elderly [1,32]. Specifically, proprioceptive deficits in the elderly are related to their general age-related decline that may impact on several sensorimotor tasks. In our study, the control group was significantly younger than stroke patients. Therefore, we cannot ascertain if the significant difference observed in the parameters' values of the two groups is due to the pathologic condition or age. Likely, both factors concurred to produce this significant difference. Hence, it is mandatory that future studies include age-matched subject groups. 


\section{CONCLUSION}

The system we developed proved able to quantitatively evaluate upper limb sense of position on the horizontal plane. It is able to distinguish healthy from pathological conditions and the measured parameters show a good reliability. This system could be employed to detect changes in sense of position of patients with sensory deficits after neuromotor rehabilitation and could enable the implementation of novel training approaches and upper limb rehabilitation protocols specifically devoted to the recovery of normal proprioception.

\section{ACKNOWLEDGEMENTS}

This research was partly funded by the " 5 per mille"-2009 project funded by the Italian Ministry of Health.

\section{CONFLICT OF INTEREST}

The authors indicated no potential conflicts of interest.

\section{REFERENCES}

[1] Goble DJ. Proprioceptive Acuity Assessment Via Joint Position Matching: From Basic Science to General Practice. Phys Ther, 2010, 90:1176-84.

[2] Goble DJ, Anguera JA. Plastic Changes in Hand Proprioception Following Force-Field Motor Learning. J Neurophysiol, 2010, 104:1213-5.

[3] Xerri C, Merzenich MM, Peterson BE, Jenkins W. Plasticity of Primary Somatosensory Cortex Paralleling Sensorimotor Skill Recovery From Stroke in Adult Monkeys. J Neurophysiol, 1998, 79:2119-48.

[4] Goodwin GM, McCloskey DI, Matthews. Proprioceptive Illusions Induced by Muscle Vibration: Contribution by Muscle Spindles to Perception? Science, 1972, 175:1382-4.

[5] Burke D, Hagbarth KE, Lšfstedt L, BG W. The responses of human muscle spindle endings to vibration of non-contracting muscles. J Physiol, 1976, 261:673-93.

[6] Roll JP, Vedel JP. Kinaesthetic role of muscle afferents in man, studied by tendon vibration and microneurography. Experimental Brain Research, 1982, 47:177-90.

[7] Wilson ET, Wong J, Gribble PL. Mapping Proprioception across a 2D Horizontal Workspace. PLoS ONE, 2010, 5:1-13.

[8] Lincoln NB, Crow JL, Jackson JM, Waters GR, Adams SA, Hodgson P. The unreliability of sensory assessments. Clinical Rehabilitation, 1991, 5:273-82.

[9] Garraway WM, Akhtar AJ, SM G, Prescott RJ. Observer Variation in the Clinical Assessment of Stroke. Age and Ageing, 1976, 5:233-40.

[10] Dukelow SP, Herter TM, Moore KD, Demers MJ, Glasgow JI, Bagg SD, et al. Quantitative Assessment of Limb Position Sense Following Stroke. Neurorehabil Neural Repair, 2010, 24:178-87.

[11] Simo LS, Ghez C, Botzer L, Scheidt RA. A quantitative and standardized robotic method for the evaluation of arm proprioception after stroke. 2011 Annual International Conference of the IEEE Engineering in Medicine and Biology Society,EMBC, 2011, 8227-8230.

[12] Squeri V, Zenzeri J, Morasso P, Basteris A. Integrating proprioceptive assessment with proprioceptive training of stroke patients. 2011 IEEE International Conference on Rehabilitation Robotics (ICORR), 2011, 1-6.

[13] Goble DJ, Noble BC, Brown SH. Where was my arm again? Memory-based matching of proprioceptive targets is enhanced by increased target presentation time. Neuroscience Letters, 2010, 481:54-8. 
[14] Goble DJ, Lewis CA, Brown SH. Upper limb asymmetries in the utilization of proprioceptive feedback. Experimental Brain Research, 2005, 168:307-11.

[15] Fuentes CT, Bastian AJ. Where Is Your Arm? Variations in Proprioception Across Space and Tasks. $J$ Neurophysiol, 2010, 103:164-71.

[16] Van Beers RJ, Sittig AC, Denier van der Gon JJ. The precision of proprioceptive position sense. Experimental Brain Research, 1998, 122:367-77.

[17] Sainburg RL, Poizner H, Ghez C. Loss of proprioception produces deficits in interjoint coordination. J Neurophysiol, 1993, 70:2136-47.

[18] Sainburg RL, Ghilardi MF, Poizner H, Ghez C. Control of limb dynamics in normal subjects and patients without proprioception. J Neurophysiol, 1995, 73:820-35.

[19] Myers JB, Guskiewicz KM, Schneider RA, Prentice WE. Proprioception and Neuromuscular Control of the Shoulder After Muscle Fatigue. J Athl Train, 1999, 34:362-7.

[20] O'Suilleabhain P, Bullard J, Dewey RB. Proprioception in Parkinson's disease is acutely depressed by dopaminergic medications. J Neurol Neurosurg Psychiatry, 2001, 71:607-10.

[21] Hospod V, Aimonetti J-M, Roll J-P, Ribot-Ciscar E. Changes in Human Muscle Spindle Sensitivity during a Proprioceptive Attention Task. J Neurosci, 2007, 27:5172-8.

[22] Wong JD, Wilson ET, Gribble PL. Spatially selective enhancement of proprioceptive acuity following motor learning. J Neurophysiol, 2011, 105:2512-21.

[23] Scott SH, Dukelow SP. Potential of robots as next-generation technology for clinical assessment of neurological disorders and upper-limb therapy. The Journal of Rehabilitation Research and Development, 2011, 48:335.

[24] Reding MJ. A model stroke classification scheme and its use in outcome research. Stroke, 1990, 21:II35-7.

[25] Shah SK. Deficits Affecting the Function of the Paralysed Arm Following Hemiplegia. Australian Occupational Therapy Journal, 1978, 25:12-9.

[26] Feys H, De Weerdt W, Nuyens G, Van De Winckel A, Selz B, Kiekens C. Predicting motor recovery of the upper limb after stroke rehabilitation: value of a clinical examination. Physiotherapy Research International, 2000, 5:1-18.

[27] De Weerdt W, Lincoln NB, Harrison MA. Prediction of arm and hand function recovery in stroke patients. International Journal of Rehabilitation Research, 1987, 10:110-2.

[28] Fortier S, Basset FA. The effects of exercise on limb proprioceptive signals. Journal of Electromyography and Kinesiology, 2012, 22:795-802.

[29] Semrau JA, Herter TM, Scott SH, Dukelow SP. Robotic Identification of Kinesthetic Deficits After Stroke. Stroke, 2013, 44(12):3414-21.

[30] Oldfield RC. The assessment and analysis of handedness: The Edinburgh inventory. Neuropsychologia, 1971, 9:97-113.

[31] Winward CE, Halligan PW, Wade DT. The Rivermead Assessment of Somatosensory Performance (RASP): standardization and reliability data. Clin Rehabil, 2002, 16:523-33.

[32] Busse M, Tyson SF. How many body locations need to be tested when assessing sensation after stroke? An investigation of redundancy in the Rivermead Assessment of Somatosensory Performance. Clin Rehabil, 2009, 23:91-5.

[33] Casadio M, Sanguineti V, Morasso PG, Arrichiello V. Braccio di Ferro: A new haptic workstation for neuromotor rehabilitation. Technology and Health Care, 2006, 14:123-42.

[34] Chen S, Cowan CFN, Grant PM. Orthogonal least squares learning algorithm for radial basis function networks. IEEE Transactions on Neural Networks, 1991, 2:302-9.

[35] Weir JP. Quantifying Test-Retest Reliability Using the Intraclass Correlation Coefficient and the SEM. Journal of Strength and Conditioning Research: The Research Journal of the NSCA, 2005, 19:231-40. 
[36] Roebroeck ME, Harlaar J, Lankhorst GJ. The Application of Generalizability Theory to Reliability Assessment: An Illustration Using Isometric Force Measurements. Phys Ther, 1993, 73:386-95.

[37] Rossetti Y, Meckler C, Prablanc C. Is there an optimal arm posture? Deterioration of finger localization precision and comfort sensation in extreme arm-joint postures. Exp Brain Res, 1994, 99:131-6.

[38] Scott SH, Loeb GE. The computation of position sense from spindles in mono- and multiarticular muscles. J Neurosci, 1994, 14:7529-40.

[39] Hall LA, McCloskey DI. Detections of movements imposed on finger, elbow and shoulder joints. $J$ Physiol, 1983, 335:519-33.

[40] Clark F, Larwood K, Davis M, Deffenbacher K. A metric for assessing acuity in positioning joints and limbs. Experimental Brain Research, 1995, 107:73-9.

[41] Clark FJ. How accurately can we perceive the positions of our limbs? Behavioural and Brain Sciences, 1992, 15:725-6. 


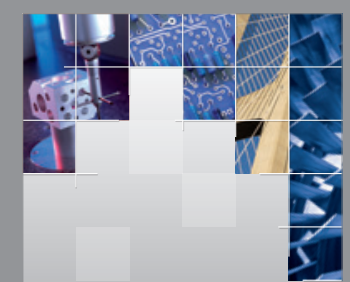

\section{Enfincering}
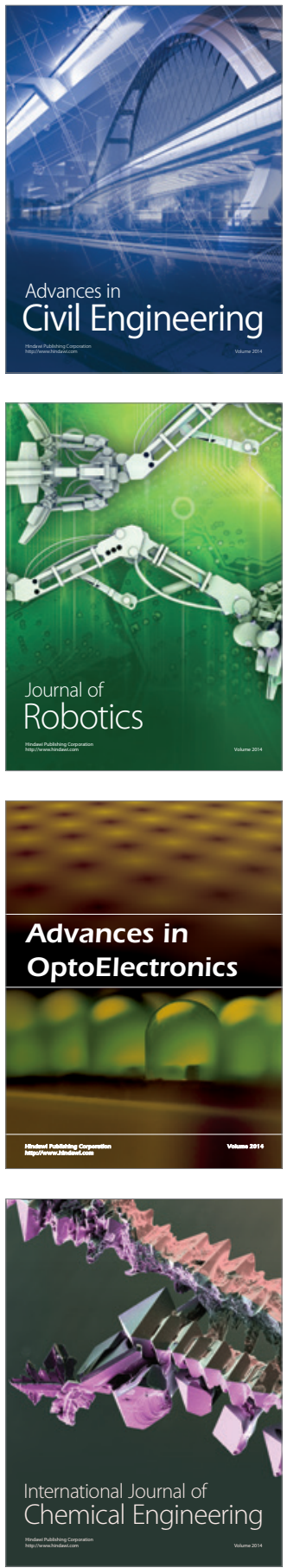

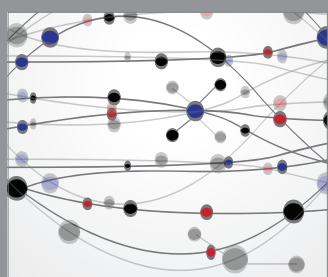

The Scientific World Journal

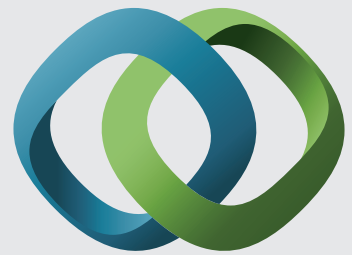

\section{Hindawi}

Submit your manuscripts at

http://www.hindawi.com
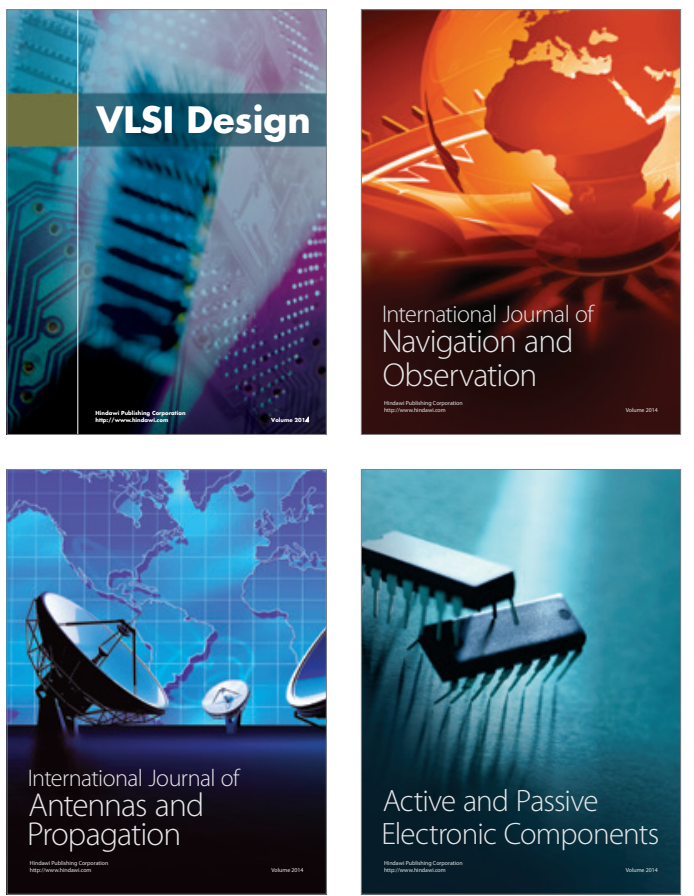
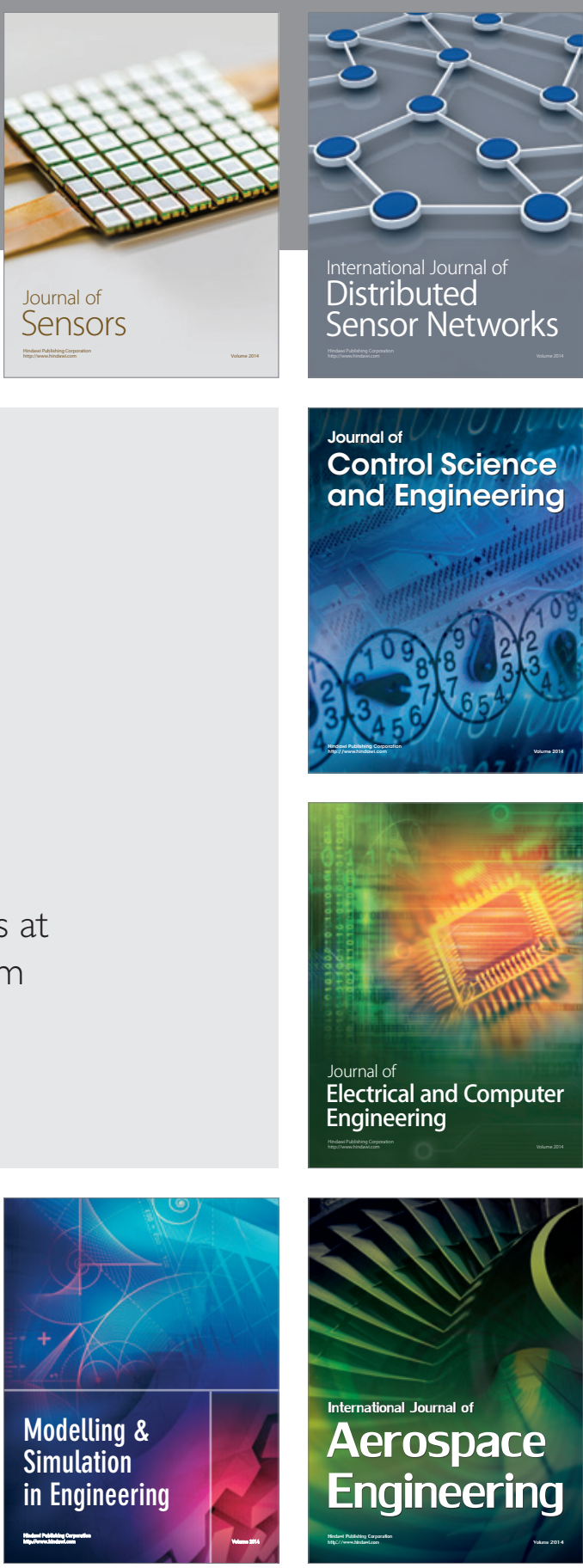

International Journal of

Distributed

Sensor Networks

Journal of

Control Science

and Engineering
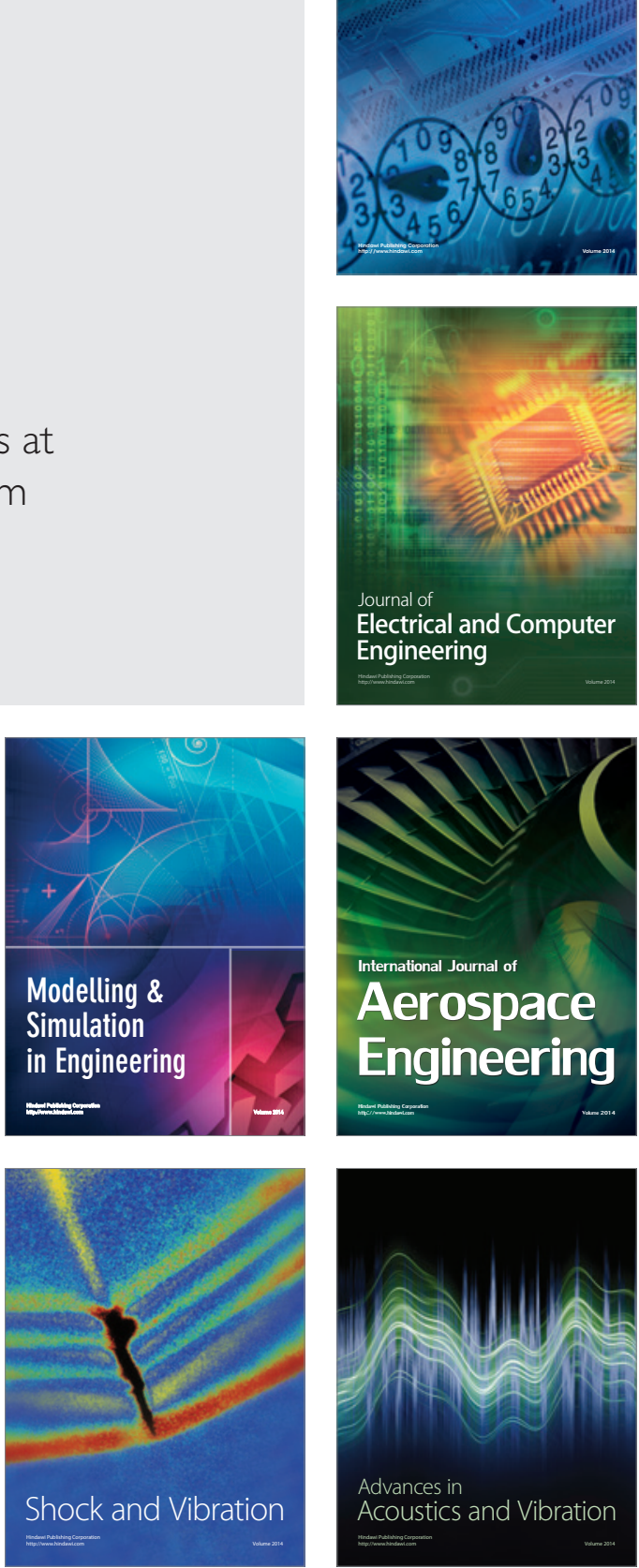